\title{
LAS DIFERENTES FUNCIONES DEL JUGAR
}

\author{
Ana Paula Lasgoity (Universidad Nacional del Nordeste)* \\ analasgoity@yahoo.com \\ Stella Maris García (Universidad Nacional de La Plata)** \\ elitagarciacitybell@gmail.com / elita@fcnym.unlp.edu.ar \\ Ada Rocío Díaz Galeano (Universidad Nacional del Nordeste) ${ }^{* * *}$ \\ rodiga7@hotmail.com
}

Recibido: 7/08/12 Aceptado: 18/11/2012

\section{Resumen}

Destacamos la importancia del juego en el Nivel Inicial, dado que funciona como escenario del mundo interno de las/os niñas/os y lo señalamos en tanto no hacemos referencia al acto sino a un proceso dinámico y complejo; permitirlo es permitir un "Espacio Transicional" entre el creer y el no creer, el adentro y el afuera. Las/os niñas/os a través del juego establecen relaciones significativas con su cuerpo, el de los otros y los objetos. Como proceso de apropiación de la cultura, a jugar se aprende y en este sentido se recupera el valor específico como función semiótica, de la imaginación, de la comunicación, de la interacción y la comprensión de la realidad.

Desde el Proyecto de Investigación "Mirada Tres" abordamos al juego como una actividad que le es grata al niño/a, hace referencia al placer que le proporciona la misma, condición primera a tener en cuenta al crear el jugar con el/la niño/a. Proponemos la denominación de jugar, marcándola con el verbo en infinitivo, para indicar su carácter de acción y para analizarla en toda su riqueza y potencialidad nos colocamos desde una perspectiva interdisciplinaria. En este artículo sistematizamos algunos parámetros teórico-conceptuales que sustentan nuestra tarea.

* Licenciada en Psicología. Universidad de la Cuenca del Plata. Profesora de la Universidad Nacional del Nordeste.

** Licenciada en Antropología. Profesora de la Universidad Nacional de La Plata.

*** Profesora en Educación Inicial; en Jardines De Infantes; en Educación Pre-Elemental Especialista en Jardines Maternales. Facultad de Humanidades Universidad Nacional del Nordeste. Doctoranda en Ciencias Cognitiva de la UNNE. 


\title{
Palabras clave
} zación.

Jugar - Nivel Educativo Inicial - Practica educativa - Socialización - Internali-

\begin{abstract}
We stress the importance of play in the initial level, because it functions as internal world stage children / as and as we pointed out we do not refer to the act but to a dynamic and complex process; permit is to allow a "transitional space" between believing and not believing, the inside and the outside. The / as children / as through it establish meaningful relationships with your body, and other objects. As a process of appropriation of culture, learned to play and in this specific value is recovered as semiotic function, imagination, communication, interaction and understanding of reality.

From the Research Project "Look Three", we approach the game as an activity that is pleasant to the child / a, refers to the pleasure it gives you the same, the first condition to be considered when creating the play with / the child / a.We propose the name of play, marking the infinitive to indicate the nature of action; for analysis in all its richness and potential we stand from an interdisciplinary perspective. This article systematize some theoretical and conceptual parameters that support our work.
\end{abstract}

\section{Key words}

Play - Initial Educational Level - Educational Practice - Socialization - Internalizing.

\section{Presentación}

"Nosotros aquí como educadores y educadoras o somos un poquito locos o no haremos nada. Si, sin embargo, fuéramos solamente locos nada haríamos tampoco. Si fuéramos solamente sanos también nada haríamos. Sólo hay un camino para hacer algo, es ser sanamente loco o locamente sano."

Paulo Freire (1970)

"Para jugar bien hay que apasionarse. Para apasionarse hay que salir del mundo de lo concreto. Salir del mundo de lo concreto es introducirse en el mundo de la locura. Del mundo de la locura, hay que saber entrar y salir. Sin introducirse en la locura no hay creatividad. Sin creatividad uno se burocratiza. Se torna hombre concreto. Repite palabras de otro."Eduardo "Tato" Pavlosky (1976)

Somos investigadores del Proyecto "Mirada Tres: la mirada y el lenguaje en el aula. Hacia la reconstrucción del conocimiento profesional docente y la enseñanza en la Educación Inicial", aprobado por la Secretaría de Ciencia y Técnica. Universidad Nacional del Nordeste. PI S.G.C. y T. UNNE - Resolución 852/11 PI: H017- 11 PERIODO 1/1/2011 Y 31/12/2015. Está inspirado en las Escuelas de Reggio Emilia, Italia, y se desarrolla en una institución de Nivel 
Inicial del mismo nombre en Resistencia, Chaco. Nos basamos en el modelo de Reggio Emilia porque el desafío que propone a los adultos que acompañamos el proceso de aprendizaje es reconocer, valorar y fortalecer la apertura al mundo de las/os niñas/os. Ellos no sólo se pueden comunicar a través del lenguaje verbal o el lenguaje gestual como otro medio habitual sino que tienen la potencialidad para utilizar los diversos códigos comunicativos y formas de pensamiento dada su posibilidad de concebir diferentes alternativas y puntos de vista de la realidad. Ante este reto los adultos aceptamos que, si bien hemos aprendido diversas formas y esquemas, sólo manejamos una perspectiva de la realidad y una forma de expresión más limitada, lo que nos coloca frente a la búsqueda reflexiva de otros modos de relación con el conocimiento más críticos, creativos y genuinos. (Holluelos,2001,2003)Las problemáticas socioculturales que involucran a la Educación Inicial se amarran al compromiso de mediar entre el YO y el TÚ en el aula de una institución educativa (Freire,1972,1970; Achilli, 2000) y los adultos-docente tenemos que lograr situarnos como referente vincular significativo, creador de sostenes, horizontes y escenarios del aprender. El análisis científico de la construcción de ámbitos adecuados para el crecimiento, desarrollo y aprendizaje de los/as niños/as de nuestra institución nos motiva a presentar sistemáticamente uno de los parámetros conceptuales que sustenta nuestra tarea cotidiana: jugar.

La hipótesis que guía esta presentación es la afirmación de que las/os niñas/os desde que nacen juegan y lo hacen en el proceso de aprender a vivir, de "ser/estar" en el mundo, de construir sus trayectorias de vida. En esta acción exteriorizan necesidades y emociones; sueñan, inventan, exploran, producen o desarman objetos; internalizan comportamientos, placenteros 0 desagradables, creencias u opiniones (Bally,1973; Amonachvili,1991) Los adultos necesariamente están/ toman /ocupan "un lugar" en ese proceso de construcción. El objetivo que nos proponemos es problematizar la acción de jugar como uno de los soportes de la socialización infantil para fundamentarla desde la perspectiva interdisciplinaria. En nuestro caso la interdisciplina alude a la articulación entre antropología, pedagogía, psicología, expresión artística y corporal en tanto cumplimentan un rol principal en la fundamentación y en la construcción de estrategias concretas para la tarea cotidiana en el aula.

\section{Construir la trayectoria de vida: el desafío de los seres humanos}

Las investigaciones del fenómeno humano, desde las ciencias sociales en general y de la antropología en particular; se han ido conformando a partir de la mirada a la diversidad y a las diferencias socioculturales, analizando los aprendizajes culturales para explicar la variabilidad de los grupos humanos en diversas épocas y espacios geográficos. Las descripciones, explicaciones y debates en los centros académicos hegemónicos hasta la década de 1960 cimentaron una matriz disciplinar con conceptualizaciones singulares respecto a 
los "otros culturales" y con la etnografía como enfoque y método de producción de conocimiento; si bien al inicio alejados de los espacios vitales de quienes serían "objeto de estudio" muy pronto el espacio propio de los investigadores sería el "teatro de operaciones" de la antropología. Debemos al Dr. Eduardo Menéndez (1967) una sistematización de las variables que conformaron esa matriz disciplinar clásica como una evidente invitación a los antropólogos argentinos y latinoamericanos a construir una disciplina que sea un instrumento para estudiar las problemáticas socioculturales propias y aportar a la transformación de nuestra conflictiva realidad. En la historia de la antropología como disciplina científica esa matriz disciplinar ha destinado espacios dispares para reflexionar sobre el juego (Enriz, 2011) y sobre las dinámicas cotidianas infantiles (Wayne, 2003). Actualmente se puede señalar que en las investigaciones antropológicas se ha constituido una arena desde donde analizar la educación infantil (Milstein et all.,2007) e investigar cualquier problemática que involucre a las/os niñas/os (Nunes,2003; Szulc,2004; Cohn,2005; Colangelo,2006; Hecht,2007) sino que se ha llegado a plantear la factibilidad de hacer etnografía con las/os niñas/ os (Milstein,2006; Podestá,2010) y no sobre ellas/os en la medida que, definitivamente, son capaces de narrar la realidad cuando se advierten habilitados para hacerlo. Subrayamos que los estudios de la niñez potencian los debates más amplios de la antropología y aportan al dialogo con otros campos de las ciencias sociales para producir conocimiento substantivo. Los estudios sobre la niñez implican reconocer a las/os niñas/os como sujetos sociales activos cuyas prácticas tienen implicancias fundamentales en las sociedades que ellas/ os mismos habitan. (Carli,1999;Rodulfo,1993)

En esta investigación seguimos los señalamientos de Berger-Luckmann (1978) cuando afirmamos que en los procesos de construcción social de la realidad, colocar la mirada en los primeros años de vida de un ser humano posibilita la sistematización de las estrategias que los diversos grupos humanos activan para convertir a ese nuevo ser en un miembro activo de la sociedad a la que se liga su pertenencia. Todo niño/a está en constante desarrollo mientras va internalizando las formas de actuar, pensar y sentir, características de su contexto sociocultural y participa en el descubrimiento y síntesis de elementos mediadores nuevos que se conjugan con el producto acumulado de la relación con generaciones anteriores. Los grupos sociales se construyen así mismos cuando van dando pertenencia al niño y se esfuerzan por enseñar los modelos socioculturales correspondientes a las posiciones que se supone ocuparán. Las formas concretas de cultura no están prefiguradas en los genes - aunque pueden determinar variaciones genéticas - y ello implica que su transmisión no se efectúa por vía de la herencia biológica, sino por la activación de los procesos de internalización, objetivación y externalización que desde algunas disciplinas se denomina aprendizaje. La manera concreta como cada grupo social organiza este aprendizaje, los valores que coloca en ellos, los contenidos que selecciona 
como principales, cuales como secundarios o son descartados, jerarquiza o denigra unos en oposición a otros, niega o esconde hechos o historias y les aparecen al niño en forma de narraciones o relatos, refranes, fábulas, leyendas, cuentos, sitios sagrados o prohibidos, rituales o costumbres. Ello se engarza en la forma como las capacidades orgánicas se ven orientadas y configuradas en los individuos concretos por el aprendizaje de su cultura y va conformando lo que se conoce por proceso de socialización Este proceso es una experiencia de construcción colectiva desde que el/la niño/a nace, parcialmente consciente y parcialmente inconsciente a través del cual se internaliza dialécticamente contenidos/forma de su grupo, de su clase social, de su sociedad y la transmite dinámicamente. Lo que le llega de sus mayores desde el nacimiento nunca es recibido pasivamente, sino que considera ese material, elige aspectos de ellos, estableciendo él mismo otras selecciones y recombina los elementos y relaciones que se le ofrecen y/o imponen; ensaya, reproduce, recrea mediante el juego significando, nominando y creando pues 'sabe' que todas las cosas tienen un nombre, dando consistencia concreta al mundo mediante su simbolización y representación. (Gili - O'Donell,1978) Cabe señalar que la socialización no iguala los comportamientos sino que, para que un grupo humano funcione como tal, es necesario que el comportamiento de sus miembros sea predecible por los que la comparten y para que la predictibilidad se cumpla se requiere equivalencia de comportamientos en algún orden concensuado.

Desde esta perspectiva, en el estudio del proceso de socialización, los investigadores científicos (antropólogos, psicólogos, pedagogos) enfatizamos la importancia de los primeros años de vida del ser humano, no sólo -como señalamos arriba- porque en este período se produce la internalización de las pautas socio-culturales que le permitirán adoptar una posición como miembro del grupo sino porque se considera que esas primeras influencias que se reciben estructuran corporal y actitudinalmente al individuo y no desaparecen, se insertan enriquecidas, modificadas, re-estructuradas en el adulto. P.Bourdieu (1991) actualiza el concepto de habitus para referirse a las estructuras que internaliza el niño y que lo estructuran, lo 'constituyen' para responder a situaciones sociales en las que se encuentra y que, a la vez, le permiten ubicarse y accionar en posteriores situaciones sociales que se le presentan. Estructuras estructurantes que actúan como estructuras estructuradas dice el autor, conceptualización que permite entender la noción de equivalencia que mencionáramos en el párrafo anterior y que resultará operativo para comprender el sentido de los comportamientos cotidianos en los cuales las acciones pasadas, lo histórico, esta entrelazado y disponible para ser reproducido o transformado.

Nuestro interés por el juego se centra en las prácticas de los sujetos, en cuanto dimensión central de producción de saber, de organización social y de construcción de perspectivas superadoras de lo cotidiano. Es decir que la acción de jugar se constituye como relevante en el proceso de construcción de 
la realidad dado que vehiculiza el hecho humano por excelencia, ser un cuerpo y tener un cuerpo. En el proceso de crecimiento las/os niñas/os son producto y a su vez productores de realidad; internalizan el mundo del que son parte, externalizan sus deseos, sus necesidades, sus inquietudes, sus fantasías y objetivan en acciones y/o productos concretos, objetos que dejan huellas y van conformando la trayectoria de sus vidas. Las experiencias de externalización, objetivación e internalización se ponen en práctica durante el juego tanto para construir su realidad subjetiva como la realidad externa a él. En este sentido, destacamos el valor de la categoría de práctica lúdica, ya que ésta enfatiza el valor de los sujetos. Hablaremos de práctica porque el juego, produce transformaciones en "los otros" a la vez que transforma al sujeto mismo, es decir, es una experiencia tanto transformadora de los sujetos como del grupo. Aquí colocamos el arte como un medio específico de conocimiento, ya que nos permite conocer, analizar e interpretar, producciones estéticamente comunicables mediante los diferentes lenguajes simbólicos (corporales, sonoros, visuales, dramáticos, literarios), y es aquí, por lo tanto, donde entran en acción los procesos de enseñar-aprender donde el lenguaje artístico actúa como comunicador y vehiculo para comunicar.Las prácticas lúdicas son experiencias formativas de los sujetos (Rockwell, 1996), en tanto constitutivas de un desarrollo activo y transformador, a la vez que condicionadas por éste mismo. Las experiencias de los sujetos son a la vez formadoras de saberes en interacción con el medio, e instancias de circulación de este saber, en las que emergen aspectos identitarios (Hoyuelos, 2004, 2009).

\section{Aprender jugando}

Las/os niñas/os - sujetos que se configuran en una complejísima trama familiar y social - miran, descubren, entienden, aprenden y aprehenden el mundo a través de su cuerpo movilizados por el "deseo", apelando a diversas formas de lenguajes desde su mundo de relaciones. Cuando hablamos de juego enmarcándonos en el proyecto pedagógico que Mirada Tres desarrolla no podemos dejar de lado las bases en las cuales se asienta el mismo, en tanto que las/os niñas/os tienen capacidades, potenciales, curiosidad e interés en construir su aprendizaje así como de comprometerse en interacciones sociales y negociar con todo lo que el ambiente les ofrece. Así es que el juego se desarrolla como punto de partida para crear un lazo social como forma de lenguaje. Es el medio por excelencia con el cual el pequeño internaliza y trasmite, expone su mundo interno y va constituyéndose como sujeto. Para construirse un cuerpo y advenir un sujeto se hace necesario un Otro que a través del lenguaje le presente al niño el cuerpo. Ese organismo vivo que nace requiere de un Otro que a través del lenguaje posibilite junto con las primeras sonrisas, reflejos, gestos, como primeras formas del jugar, lograr un cuerpo subjetivado. Diríamos: anudando el lenguaje en ése que va siendo de a poco un cuerpo. 
Pensamos a cada niña/o no aisladamente, sino, en conjunto con otros niñas/os, con la familia, con los docentes, con el ambiente escolar, con la comunidad y el resto de la sociedad. "(...) El juego humano resulta de un complejo conjunto de significaciones biológicas y psicológicas subjetivas al cual se le superpone un cuadro de significaciones relacionadas con el proceso de identificación intersubjetiva y social con el otro humano" (Gómez, 2004:41).Es allí en el juego donde el/la pequeño/a se muestra como co-constructor, desde el comienzo de su vida, de conocimiento, cultura y de su propia identidad. Es entendido y reconocido como miembro activo de la sociedad. Ahora la pregunta es: ¿Cómo?, ¿Cómo logra este proceso? ¿Cómo conoce? ¿Cómo se apropia de sí y los demás?, por medio del juego, juego que propone un sujeto activo, que construye el conocimiento y crea por medio de él los significados del mundo. La/ el niña/o extrae de la experiencia los modelos de acción que intentará generalizar y que utilizará para modelar la realidad. Estas adquisiciones se resuelven en el juego del equilibrio entre asimilación y acomodación. Es así que, en este caso, la principal función psicológica del juego en la infancia está dada por el hecho de que permite a los/as niños/as asimilar las estructuras complejas de la realidad a las posibilidades de comprensión y acción de sus cuerpos.

Desde lo pedagógico tomamos a la escucha como una táctica fundamental de este proyecto Mirada Tres. El diálogo democrático implica la capacidad de escuchar y la recíproca expectación como cualidad importante que activa la comunicación y el intercambio reciproco; esta acción requiere ser sostenida, entendida, documentada. El autoaprendizaje de las/os niñas/os, el hecho de que el grupo se vuelva consciente de ser "un lugar de enseñanzas", un lugar donde los lenguajes son enriquecidos, multiplicados, refinados, generados e interpretados en sus procesos intrínsecos que como un juego van haciendo huella, marca, para dar cuenta de una constitución subjetiva única e irrepetible. En ese documentar entonces son visibles los tipos de juego, o mejor dicho los diferentes lenguajes conforman niños/as únicos e irrepetibles.

Es aquí donde la familia ocupa un rol trascendental orientando los procesos de aprendizaje y activando, donando elementos necesarios para ello, como el aspecto psicológico y la continuidad de lo biológico. El adulto ofrece el "sentido del espacio" para ayudar al niño y a la niña a comprenderse y comprender sus acciones a través del reconocimiento. Introducir a los niños en espacios nuevos, sugerentes y llenos de posibilidades es un estímulo para el juego simbólico y la acción para los discursos narrativos en los que su atención se fija y en los que ellos se convierten en los protagonistas dentro de una historia totalmente nueva.

$\mathrm{El} / \mathrm{la}$ pequeño/a es estimulado/a por estos padres en una primera y estrecha relación con la figura materna para luego, a modo de corte, incluir un tercero referente significativo de la triada. Así los modelos identificatorios van cobrando reveladora importancia y la apropiación de un espacio subjetivo posibilita la separación o independencia familiar. La experiencia de juego es notable como 
actividad que remite a la posibilidad de obtener recursos elaborativos y a su papel en la realización de deseos. Vemos el juego como una vía para expresar deseos y descargar sentimientos por lo que se constituye en pilar del equilibrio emocional. Es en ese 'Espacio Transicional' que se ubica el mundo interno y la realidad, dando cabida al juego creativo y a la imaginación permitiendo al niño/a resolver sus conflictos internos así como también experimentar y descubrir su entorno. Fundamentalmente es un medio para la estructuración del lenguaje y el pensamiento. La zona intermedia entre la seguridad materna y la incertidumbre de un medio (al menos desconocido), deben ser articuladas por un lazo común que puede tomar según Winnicott (1989: 145) la forma de un objeto transicional.

"La experiencia del bebé con esta confiabilidad a lo largo de un período hace nacer en él, y en el niño en crecimiento, un sentimiento de confianza. La fe del bebé en la confiabilidad de la madre, y por lo tanto en la de otras personas y cosas, permite la separación del noyo y el yo. Pero al mismo tiempo se puede decir que la separación se evita al llenar el espacio potencial con juegos creadores, con el empleo de símbolos y con todo lo que a la larga equivale a una vida cultural." (Winnicott, 1989: 145)

A partir del juego del/la niño/a vemos como se va estructurando la identidad, el dominio de las estructuras del lenguaje, los códigos y la producción de sentido que constituye el eje de los encadenamientos funcionales del lenguaje, mediados por los códigos lingüísticos y la simbología popular de los cuales se apropian los sujetos para caracterizar sus prácticas sociales y culturales. A partir del juego entonces se irá constituyendo la identidad social (o de grupo), a través de los códigos que funcionan convencionalmente, desde el interior hacia fuera. Los diferentes tipos de juegos, simbólicos, de reglas, entre otros, son la base fundante para la identificación con el otro. Se intercambia, se trasforma y reproduce significados fundamental para el proceso de identificación. "La conciencia de sí mismo y la conciencia del mundo, se desarrollan producto de las relaciones que el niño traba con el mundo, fundadas en la acción deliberada, en el deseo de hacer, confrontando a la realidad física y social" (Freire, 1972, p.33).

Asimismo el juego es un derecho y una característica singular de los/as niños/as. El juego y las experiencias globalizadoras representan los ejes del modelo didáctico para la construcción de las propuestas de enseñanza en la Educación Inicial y base del proyecto que aquí nos convoca. Las propuestas diseñadas en torno de ello deben adecuarse a la heterogeneidad de los sujetos y sus contextos. El espacio escolar es depositario del imaginario colectivo y traductor de dimensiones simbólicas y pedagógicas y tiene influencia en la percepción y representación que las personas hacen de él. 
Mirada Tres es un espacio para hacer y deshacer, para crear, para descubrir, para adquirir nuevas habilidades. El juego debe ser entendido en sus diversas significaciones. El juego no es ajeno al proyecto pedagógico, el juego es un contenido, un eje trasversal y no puede ser aislado debe tener conceptualmente coherencia con este proyecto. Es por ello que nos proponemos en cada ocasión diseñar e implementar estrategias adecuadas a los contextos particulares que planificamos en cada tarea cotidiana.

Al igual que el valor educativo de la improvisación ponderamos el de la creación en la confección de juguetes, por ejemplo, esto es, la construcción de objetos de juego. La tarea cotidiana pone en evidencia el ejercicio activo de la imaginación de los/as niños/as cuando elaboran con materiales de desecho, descartables, sus propios elementos para jugar, coches hechos con cajas de zapatos pintadas, muñecos con bolsas o palitos, etc; dejamos en un segundo plano los juegos estereotipados y comerciales que nada tienen que ver con ellos y que el mercado impulsa a consumir.

Lo propio de las infancias es el jugar, esta es su ocupación favorita, como espejo que anuda lo real lo simbólico, lo imaginado, la fantasía. En este jugar es necesario el toque y la palabra del Otro para que el cuerpo se amarre con el universo discursivo. Los niños son sujetos deseantes y, ante el desafío impuesto por la práctica, supone el persistente ejercicio de extender los límites que la disciplina impone para abocarnos a la realidad de los/as niños/as de manera más creativa, más genuina y significativa.

El equipo de profesionales de Mirada Tres está convencido que el jugar permite responder a la demanda genuina de los/as niños/as, para lo cual concebimos los talleres de juego creativos con un encuadre de tiempo y espacio, que toma en cuenta la singularidad, espacio donde se pone en práctica el pintar, el manchar y mancharse, el relato de un cuento, el juego en sí mismo, la producción de sonidos musicales, la dramatización teatral. En simultaneidad con los talleres a los/as niños/as, los padres trabajan en el taller de padres donde se intenta comprender y acompañar a sus hijos/as.

Vassily Kandinsky, pintor nacido en Moscú en 1866, proclama la ley de la necesidad interior, con el consiguiente abandono progresivo del mundo visible. Freud alude a "la necesidad subjetiva de lo fantástico", él nos dice que son los momentos privilegiados en que el artista mira en lo desconocido, imagina lo oculto y vislumbra lo visible. El juego de fantasía y realidad, conjunción entre realidades, exterior y psíquica. Manifestar lo bello, lo sagrado, el encantamiento, la relación con un mundo invisible y, hacer soportable un real insoportable es imposible sin metáfora.

Maud Mannoni (1983) expresa "Es necesario un territorio de juego para que la imaginación tenga derecho a la ciudadanía”.Podríamos decir, entonces, que el jugar es producto de la cultura, afirmando que se aprende y, en este sentido, se recupera el valor íntimo que tiene para el desarrollo de las posibili- 
dades representativas, de la imaginación, de la comunicación y la comprensión de la realidad.

\section{A modo de conclusión}

Iniciamos esta presentación con la afirmación de que las/os niñas/os desde que nacen juegan y lo hacen en el proceso de aprender a vivir, de "ser/estar" en el mundo, de construir sus trayectorias de vida y desarrollamos, desde la perspectiva interdisciplinaria que construimos, las diferentes funciones del jugar en las trayectorias de socialización. Apoyándonos en nuestra experiencia y en los numerosos autores que reivindican la dinámica de trabajo en las escuelas de Reggio Emilia subrayamos los aportes del juego en todo proyecto pedagógico sin embargo no pasamos por alto que en las administraciones, a través de leyes y decretos, y peor aun, en la historia de la las Ciencias de la Educación, el juego ha tenido mala prensa. Se ha clasificado al juego como una ocupación perniciosa proponiendo se lo reduzca a la mínima expresión y se ocupe a niñas/ os con matemática, lectoescritura, informática, inglés.

... hay clases en las que la mayor parte del tiempo los niños están sentados haciendo fichas, algo que va contra la naturaleza de estas edades. Maestros y maestras gritonas que asustan a los niños -quienes, si algo necesitan en estas edades, es sobre todo seguridad-, profesores y profesoras que miran el reloj pues las horas se les hacen eternas". De esta forma, el currículo escolar de la Educación Infantil está robando en un $90 \%$ el tiempo de juego para los niños. Tiempo que las criaturas necesitan vitalmente para crecer y construir su cultura. El juego, en estas situaciones, es visto como una pérdida de tiempo. Sólo se permite jugar cuando se han terminado las actividades curriculares que se consideran jerárquicamente importantes (Hoyuelos 2009, pp. 59-60)

Paralelamente vale señalar la connivencia de posturas de profesionales, editoriales y empresas que afirman que el niño sólo aprende jugando; generando como respuesta la invención de pseudo-juegos y juguetes didácticos que, desnaturalizando el auténtico concepto de lo lúdico, estructuran elementos según objetivos didácticos marcados de antemano. "De este modo el mercado neoliberal se ha llenado de materiales para enseñar números, letras, creatividad, asociaciones, seriaciones, etc. "Y una vez más el aplastante poder del adulto se ha impuesto sobre la cultura infantil; soslayando que el juego es aventura, incertidumbre, azar, sueño, viaje a lo desconocido, virtualidad" (op.cit,69).

El proyecto Mirada Tres propone convertir las aulas en escenarios lúdicos (con materiales, generalmente no estructurados y estéticas apropiadas) donde los/as niños/as y niñas pueden inventar sus juegos y consolidar nuestra tarea docente en el aprender día a día junto con los niños y niñas. Hemos decidido 
disfrutar de ver cómo los niños y niñas juegan juntos y aprenden cosas inimaginables antes de "esa disponibilidad emocional y cognitiva de escucha: "lo verdaderamente importante”, según subraya Hoyuelos $(2009,70)$.

\section{Referencias Bibliográficas}

- $\quad$ Aberastury, A. (1976). El niño y sus juegos. Buenos Aires: Paidós.

- Achilli E. (2000). "Contexto y cotidianeidad escolar fragmentada". En: Cuadernos de Antropología Socia $\mathrm{N}^{\circ} 12$, Buenos Aires: FFyL UBA. Pp.: 11-30

- Amonachvili, C. (1991). Un impulso vital. En: El Juego Revista El Correo de la UNESCO, Francia.

- Bally, G. (1973). El juego como expresión de la libertad; Mexico: Fondo de Cultura Económica,

- Berger, P. y Luckmann, T. (1972). La construcción social de la realidad; Buenos Aires: Amorrortu.

- $\quad$ Bourdieu P. (1991). El Sentido Práctico. Barcelona: Editorial Taurus Humanidades.

- Carli, S. (1999). "La infancia como construcción social". En Carli, S. (comp.) (1991) De la familia a la escuela. Infancia, socialización y subjetividad, Buenos Aires: Santillana.

- Cohn, C. (2005). Antropologia da Criança. Rio de Janeiro: Jorge ZAHAR Editor.

- Colangelo, A. (2006). "La crianza en disputa. Un análisis del saber médico sobre el cuidado infantil". En: VIII Congreso Argentino de Antropología Social, Universidad Nacional de Salta.

- Dal Santo, L. (1997). El Taller de educadores. Una alternativa de investigación del lugar del juego en el contexto escolar. En: Varios Autores Juego, Aprendizaje y Educación. Revista ALTERNATIVAS, № 8. Serie: Espacio Pedagógico. Facultad de Ciencias Humanas, Universidad Nacional de San Luis, R. A.

- De Bono, E. (1987). El pensamiento lateral. Buenos Aires: Paidós.

- Enriz, N. (2011). "Antropología y Juego: Apuntes para una reflexión". En: Cuadernos de Antropología, №34, Jul/Dic Buenos Aires: FFYL.

- Fernández, A. (1989). La Inteligencia Atrapada. Buenos Aires: Ed. Nueva Visión.

- Freire, P. (1972). Educación como práctica de la Libertad. Buenos Aires: Siglo XXI.

- Freire, P. (1970). Pedagogía del oprimido. Montevideo: Ed. Tierra Nueva.

- $\quad$ Freud, S. (1968). Obras Completas. (II) 1 Madrid: Biblioteca Nueva. Editorial Luis López Ballesteros y de Torres.

- Gili, E., O’ Donell, P. (1978). El juego. Técnicas lúdicas en Psicoterapia Grupal de Adultos. Barcelona: Gedisa.

- Giordan, A.; de VECCHI, G. (1995) Obstáculos en la apropiación del saber. En: Los orígenes del saber. De las concepciones personales a los conceptos científicos. Sevilla: Díada Editora, 2da. Edición.

- Hecht, A.C. (2007). "Reflexiones sobre una experiencia de investigación - acción con niños Indígenas. Napaxaguenaxaqui na qom llalaqpi da ỹiyiñi na l'aqtac". En: Boletín de Lingüística 28 (19): 46-65, Buenos Aires:FFYL.

- Hoyuelos, A. (2001). El pensamiento y obra pedagógica de Loris Malaguzzi y su repercusión en la Educación Infantil. Tesis doctoral. Universidad Pública de Navarra

- Hoyuelos, A. (2003). La complejidad en el pensamiento y obra pedagógica de Loris Malaguzzi. México: Multimedios, Col. Pensamiento Pedagógico. 
- Hoyuelos, A. (2004). La Ética en el pensamiento y obra pedagógica de Loris Malaguzzi. Barcelona: Octaedro-Rosa Sensat.

- Hoyuelos, A. (2009). Ir y descender a y desde Reggio Emilia.CEE Participación Educativa, 12, pp. 171-181

- Jaitín de Langer, R. (1985) Aprendizaje, Juego y Placer. Buenos Aires: Ed. Búsqueda.

- $\quad$ Litwin, E. (1999). El campo de la didáctica: la búsqueda de una nueva agenda. En: Varios Autores Corrientes didácticas contemporáneas. Buenos Aires: Paidós, 3ra.Reimpresión.

- $\quad$ Mantilla G., L. (2000). Juego y jugar como experiencias separadas: la utilidad práctica de esta distinción. Comprensión de la experiencia lúdica adulta En: Ramos Ramírez y Martínez Martínez (Coord) Diversas miradas sobre el juego. México: Tierra Firme.

- Mauriras-Bousquet, M. (1991). Un oasis de dicha. En: El Juego, Revista El Correo de la UNESCO, Francia.

- Mannoni, M. (1983). La educación imposible. México: Siglo XXI.

- Menendez, E. (1967) "El modelo antropológico clásico". Mimeografiado. Bs As.

- Milstein, D. (2006). "Y los niños, ¿por qué no? Algunas reflexiones sobre un trabajo de campo con niños". En: Revista Avá N 11 Pp.: 49-59. Misiones: Programa de Antropología Social, Universidad Nacional de Misiones.

- $\quad$ Milstein, D., Fernandez, M., Garcia, A., Garcia, S. y Paladino, M. (2007). "Panorama de la Antropología y la Educación escolar en la Argentina 1982-2006“ En Anuario de Estudios en Antropología Social 2006 Pp. 77-96.Buenos Aires: Centro de Antropología Social - IDES Agosto

- Nunes, A. (2003). Brincando de ser criança, contribuçoes da etnología brasileira à antropología da infancia. Tese de Doutorado. Universidade de São Paulo.

- Pavlovsky, E. (1976). Reflexiones sobre el proceso creador/El señor Galíndez. Buenos Aires: Editorial Proteo.

- $\quad$ Pasel, S. (1991) Aula - Taller. Buenos Aires: Aique, Citado en Caracterización general de las distintas unidades curriculares. LINEAMIENTOS CURRICULARES (2001) de la Formación Docente para Nivel Inicial. Dirección General de Educación Superior, Dirección General de Planeamiento, Dirección de Currícula. Secretaría de Educación, Gobierno de la Ciudad de Bs. As.

- $\quad$ Piaget, J. (1973). Psicología de la inteligencia. Buenos Aires: Psique.

- Porlán, R. (1993). El ser humano como científico: una metáfora sobre la epistemología constructivista del conocimiento cotidiano. En: Constructivismo y escuela. Sevilla: Díada Editora,

- $\quad$ NIÑOS Y NIÑAS NAHUAS - Podesta Siri, R. (2010) Nuestros pueblos de hoy y siempre. El mundo de los niños y niñas nahuas de México a través de sus propias letras y dibujos. México: Universidad de Puebla.

- $\quad$ Rockwell, E. (1996). De huellas, bardas y veredas: una historia cotidiana en la escuela. En: La escuela cotidiana. México: Fondo de Cultura Económica.

- $\quad$ Rodulfo R. (1993) El niño y el significante. Buenos Aires: Paidos.

- Szulc, A. (2004). "La antropología frente a los niños: De la omisión a las "culturas infantiles". En: VII Congreso Argentino de Antropología Social, Universidad Nacional de Córdoba, Villa Giardino, Córdoba.

- Winnicott, D. W. (1971) Realidad y juego. 2 ed. Tr. Floreal Mazía. Barcelona: Gedisa.

- "NúCleOS DE APRENDIZAJES PRIORITARIO"- (2004) Buenos Aires: Ministerio de Educación, Ciencias y Tecnología de la Nación.

- Wayne, R. (2003). Un paseo por la Antropología Educativa. En Revista Nueva Antropología, págs 10-28. México. 